\title{
Atlas computarizado para la planificación de neurocirugías estereotácticas funcio- nales guiadas por imágenes
}

\author{
M. Carballo-Barreda; R. Rodríguez-Rojas; A. Torres-Montoya y G. López-Flores
}

Grupo de Procesamiento de Imágenes y Servicio de Neurocirugía. Centro Internacional de Restauración Neurológica. Ciudad de la Habana. Cuba.

\section{Resumen}

Objetivo. Desarrollar un atlas estereotáctico computarizado de Schaltenbrand y Wahren para la planificación de neurocirugías funcionales guiadas por imágenes. Este atlas está integrado al sistema de planificación STASSIS, desarrollado para computadoras personales.

Métodos. Los cortes originales del atlas fueron digitalizados y se trazaron los contornos para ambos hemisferios. El corregistro del atlas con las imágenes del paciente se realiza mediante transformaciones rígidas y no rígidas. Se presentan las herramientas computacionales implementadas y sus aplicaciones durante el procedimiento quirúrgico.

Resultados. El sistema permite la navegación continua tanto a través de los cortes originales como de las reconstrucciones, la sincronización de múltiples vistas y el uso de ampliaciones, lo que permite incrementar la exactitud en la localización de las comisuras y los blancos quirúrgicos, así como la selección óptima de las trayectorias. Los contornos del atlas se pueden ajustar a la anatomía del paciente. La posición del atlas en la región del blanco se puede actualizar de forma interactiva $y$ simular la posición y volumen de las lesiones.

Conclusiones. Los beneficios de esta metodología incluyen la mejora en la exactitud de la localización del primer blanco quirúrgico, disminución del número de trayectos con los electrodos y, por tanto, del tiempo de la cirugía, y la reducción de las posibles complicaciones quirúrgicas.

PALABRAS CLAVE: Atlas cerebral. Estereotaxia. Neurocirugía funcional. Sistema de planeamiento.

Computerized atlas for image-guided stereotactic functional neurosurgery

\section{Summary}

Objective. A computerized version of the Schaltenbrand

Recibido: 15-01-07. Aceptado: 25-02-07 and Wahren's stereotactic brain atlas for image-guided functional neurosurgery planning has been developed and integrated into our PC-based planning system.

Methods. The SW atlas plates were digitized, contoured and labeled for both hemispheres. The computerized atlas may be interactively registered with patient's data using linear and non-linear transformation. The implemented computational tools and applications are presented.

Results. Our computer system permits navigation through original or reconstructed slices, multiple-views synchronization and zoom to improve the localization of the commisures and the surgical targets, likewise the optimum path selection. Atlas position in the target's region can be interactively actualized and lesion's position and volume may be simulated.

Conclusions. Its benefits of this approach include increased accuracy of target definition, decreased the number of electrode tracts and for instance the time of the surgery, and reduced surgical complications.

KEY WORDS: Brain atlas. Stereotaxy. Functional neurosurgery. Planning system.

Introducción

A pesar del avance en las técnicas de adquisición de imágenes médicas, el proceso de localización de los blancos quirúrgicos en la neurocirugía funcional sigue siendo complejo, y se basa esencialmente en la experiencia del equipo que realiza la cirugía. Las imágenes de Tomografía Axial Computarizada (TAC) no ofrecen el detalle anatómico necesario para reconocer entre estructuras cerebrales con poco contraste físico (en términos de absorción de los rayos $\mathrm{X}$ ) con el tejido circundante, como es el caso de los ganglios basales. Las imágenes de Resonancia Magnética Nuclear (RMN) poseen mejor contraste anatómico que las de TAC para tejidos blandos, pero generalmente están afectadas por distorsiones geométricas, lo que puede afectar significativamente la exactitud en la localización de los blancos ${ }^{8,16,18,24}$. El uso de marcos estereotácticos introduce artefactos debidos a cambios bruscos en la susceptibilidad 


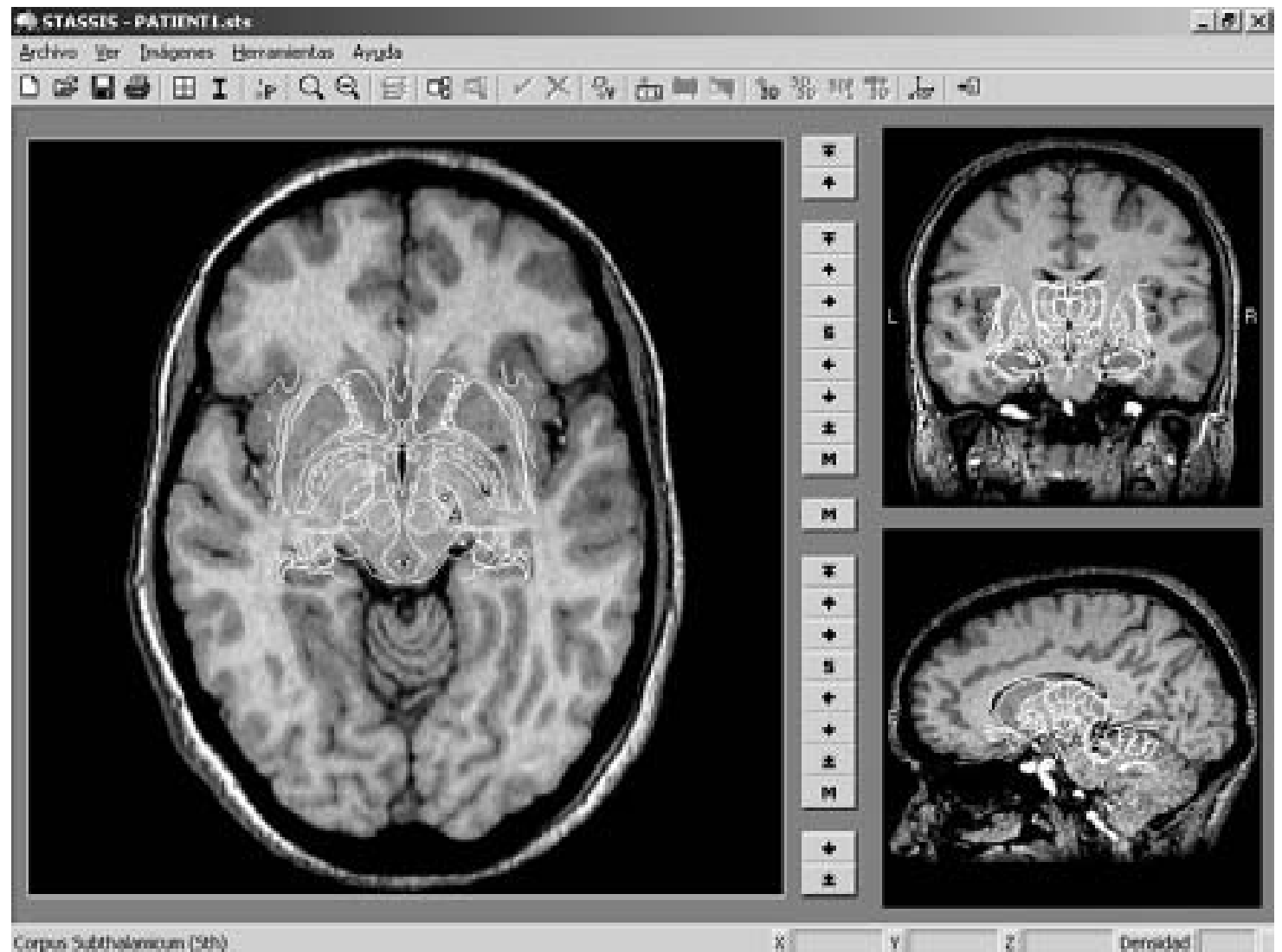

Figura 1. Interfaz del sistema STASSIS donde se observan las tres series del atlas digital de Schaltenbrand \&Wahren superpuestas a sus respectivos cortes anatómicos.

magnética ${ }^{8,16,24}$, lo que contribuye al incremento de las distorsiones geométricas. A esto se agrega que los sistemas de RMN estándar aún tienen limitaciones para la diferenciación exacta de los bordes anatómicos de los ganglios basales.

Una alternativa para incrementar la exactitud en la localización de los blancos quirúrgicos, es obtener información anatómica a partir de un atlas digitalizado adaptado

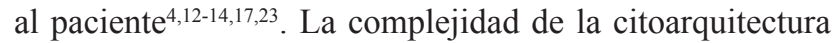
cerebral y la variabilidad inter-sujetos han hecho de los atlas una herramienta indispensable para manipular, analizar e interpretar la información imagenológica en el proceso pre e intra-operatorio.

En el presente trabajo presentamos un atlas estereotáctico digitalizado, basado en el atlas de Schaltenbrand y Wahren $(\mathrm{SW})^{15}$, para ser usado en la planificación pre e intra-operatoria de cirugías funcionales para trastornos del movimiento. Este atlas fue integrado en un sistema computarizado para la planificación de cirugías estereotácticas, desarrollado en el Centro Internacional de Restauración Neurológica (CIREN) ${ }^{4}$. Se presentan y discuten las herramientas computacionales implementadas, sus aplicaciones en diferentes etapas del procedimiento quirúr- gico, así como las ventajas y desventajas del uso del atlas electrónico de SW.

\section{Métodos}

\section{Digitalización y visualización}

Los 57 cortes de las series horizontal, frontal y sagital del atlas $\mathrm{SW}^{15}$, previamente digitalizados y vectorizados, fueron integrados al sistema de planificación STASSIS (CIREN, Habana, Cuba), el cual permite la planificación de múltiples aplicaciones neuroquirúrgicas guiadas por imágenes. Las imágenes de TAC o RMN del paciente se muestran conjuntamente con la información correspondiente de los contornos de las estructuras presentes en el atlas. Este procedimiento ha sido implementado para los cortes axiales, coronales y sagitales (figura 1). Al señalar una estructura se reporta su nombre, así como la identificación del corte del atlas que es visualizado.

\section{Metodología de planificación}

La metodología para la realización de neurocirugías 


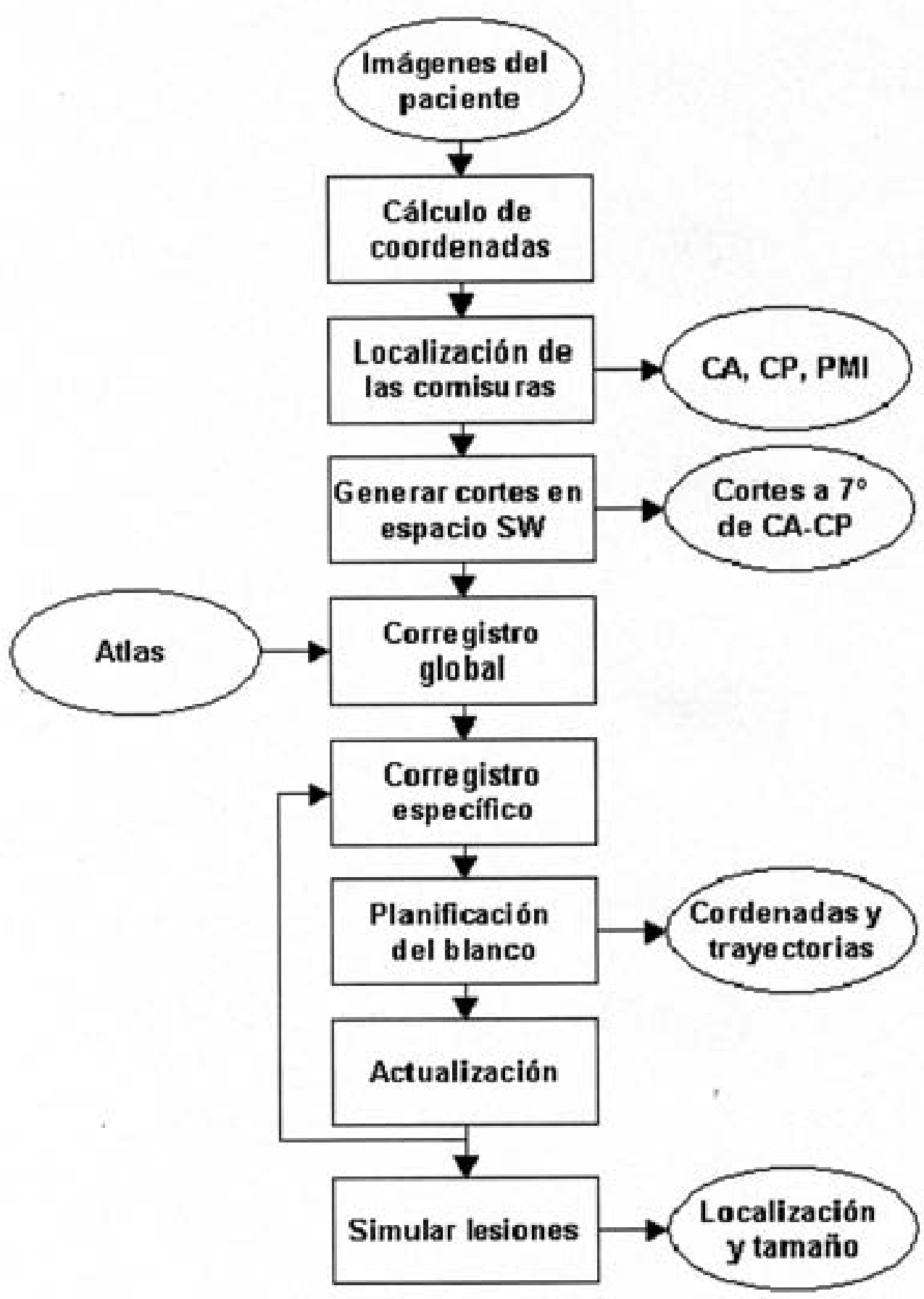

Figura 2. Metodología para la realización de neurocirugías funcionales utilizando el programa STASSIS. Los rectángulos representan operaciones y los óvalos representan datos. (Comisura Anterior-CA, Comisura Posterior -CP, Punto Medio Intercomisural -PMI, Schaltenbrand \&Wahren -SW).

funcionales, utilizando el atlas digitalizado SW, se muestra en el diagrama de flujo de la figura 2. A continuación se describen los pasos para realizar estos procedimientos con el programa STASSIS.

Cargar imágenes. El sistema STASSIS permite cargar imágenes en formato DICOM (del inglés Digital Imaging and Communication in Medicine) $)^{1} \mathrm{o}$ en el formato propio del sistema. Las planificaciones son salvadas en un formato interno, en el cual se almacenan conjuntamente con las imágenes utilizadas para la planificación, las obtenidas 
mediante reconstrucciones, así como la información de los blancos, trayectos, lesiones y cualquier otro elemento de la planificación quirúrgica. Es posible generar, en tiempo real, reconstrucciones axiales, coronales y sagitales o en cualquier dirección arbitraria para facilitar la ubicación espacial de las diferentes estructuras. Cualquiera de estas reconstrucciones puede visualizarse en la vista principal e intercambiarse para su manipulación (Figura 1).

Cálculo de coordenadas. Para el cálculo de coordenadas creamos un método vectorial, basado en la descripción analítica de todas las posibles relaciones geométricas existentes en la posición de los marcadores de referencia'. Son calculadas las coordenadas 3D de tres puntos no alineados de la imagen, a partir de las cuales es posible obtener la matriz de transformación de coordenadas estereotácticas a coordenadas de la imagen. De esta forma, para cualquier punto visible en las imágenes podemos obtener sus coordenadas estereotácticas. Dada la generalidad de este método puede ser utilizado eficientemente para diferentes marcos estereotácticos. En nuestro sistema de planificación ha sido implementado y comprobado el cálculo de coordenadas para los marcos estereotácticos Leksell-G, Estereoflex, Riechert-Mundinger y Micromar. El sistema STASSIS permite además la manipulación de imágenes sin marco, lo que facilita el uso de imágenes provenientes de diversas fuentes durante el acto quirúrgico y en el análisis postoperatorio.

Localización de las comisuras. La exactitud en la localización de la comisura anterior (CA) y comisura posterior (CP) define el posicionamiento correcto del atlas con respecto a la anatomía real del paciente. La posición de las comisuras puede ser verificada de forma tridimensional con las facilidades del sistema. La visualización del plano intercomisural puede mejorar la certeza en la localización de las comisuras, por lo que, en los casos en que CA y CP no estén en el mismo plano axial, es conveniente reconstruir las imágenes con esta orientación.

Generar cortes en espacio de Schaltenbrand y Wharen. $\mathrm{El}$ atlas SW original consiste en tres secciones definidas por CA y CP. La serie axial está orientada con $7^{\circ}$ de inclinación con respecto al plano inter-comisural, mientras las series frontal y sagital son perpendiculares a aquél y entre ellas ${ }^{15}$. Para visualizar los contornos del atlas digital, superpuestos a los cortes anatómicos, es necesario hacer reconstrucciones con los ángulos descritos.

Corregistro global. El corregistro inicial del atlas digitalizado con la anatomía del paciente se realiza aplicando una técnica de escalamiento lineal, basada en la distancia entre CA y CP, y el ángulo del plano CA-CP.

Corregistro especifico. La adaptación del atlas a la anatomía del paciente se puede refinar de forma interactiva, mediante el corregistro de las estructuras del atlas con las estructuras correspondientes visibles en el conjunto de imá- genes del paciente. El corregistro del atlas con la anatomía real se realiza a través de transformaciones geométricas rígidas y no rígidas, las cuales describen el mapeo punto a punto del atlas al cerebro individual. Los parámetros de transformación rígidos incluyen la traslación en los tres ejes coordenados. En general a cualquier punto $\mathbf{p}(\mathrm{x}, \mathrm{y}, \mathrm{z}) \mathrm{se}$ le puede aplicar una traslación en $\mathbf{q}$ unidades, de tal forma que la transformación en el punto $\mathbf{p}^{\prime}\left(\mathrm{x}^{\prime}, \mathrm{y}^{\prime}, \mathrm{z}^{\prime}\right)$ queda definida por:

$$
\mathbf{p}^{\prime}=\mathbf{p}+\mathbf{q}
$$

En términos matriciales, la traslación se puede expresar de la forma:

$$
\left.\left[\begin{array}{c}
x^{\prime} \\
y^{\prime} \\
z \\
1
\end{array}\right]=\left[\begin{array}{lllc}
1 & 0 & 0 & q_{1} \\
0 & 1 & 0 & q_{2} \\
0 & 0 & 1 & q_{3} \\
0 & 0 & 0 & 1
\end{array}\right] \begin{array}{c}
x \\
y \\
z \\
1
\end{array}\right]
$$

donde $q 1, q 2, q 3$, son los parámetros de la transformación. Las transformaciones no rígidas representan el escalamiento lineal de las estructuras del atlas en los tres ejes ortogonales, el cual puede representarse de la forma:

$$
\left[\begin{array}{l}
x^{\prime} \\
y^{\prime} \\
z^{\prime} \\
1
\end{array}\right]=\left[\begin{array}{cccc}
q_{1} & 0 & 0 & 0 \\
0 & q_{2} & 0 & 0 \\
0 & 0 & q_{3} & 0 \\
0 & 0 & 0 & 1
\end{array}\right]\left[\begin{array}{l}
x \\
y \\
z \\
1
\end{array}\right]
$$

Los parámetros de transformación qn pueden ser modificados explícitamente en cualquier momento durante el acto quirúrgico (Figura 3). En los casos en que la posición

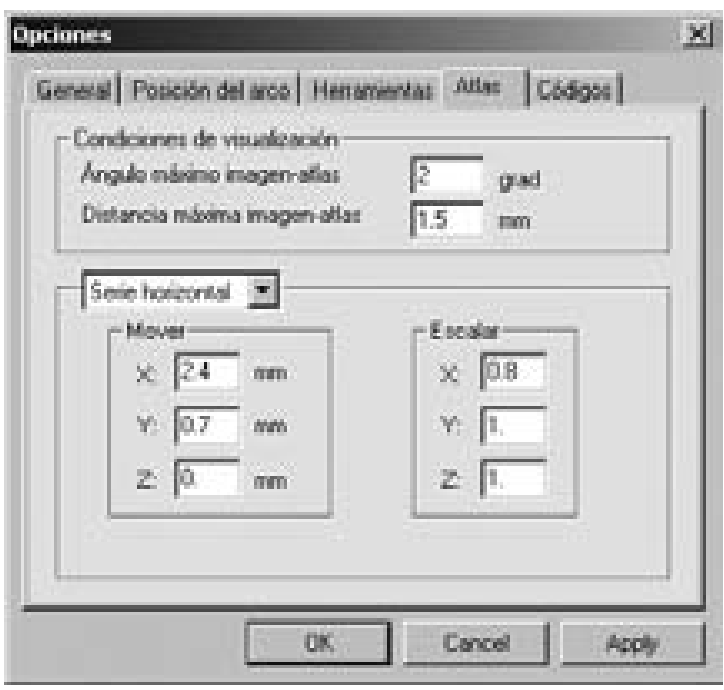

Figura 3. Parámetros modificables para la transformación: traslación ('Mover') y escalamiento ('Escalar'). También es posible modificar los parámetros 'Distancia máxima imagen-atlas' y 'Ángulo máximo imagen-atlas'. 
Figura 4. Utilización del atlas computarizado en diferentes etapas de la planificación quirúrgica. A) Corregistro global del atlas con respecto a las comisuras, $B$ ) corregistro especifico y localización del primer blanco, C) actualización a partir de la información neurofisiológica y simulación de las lesiones y D) evaluación postoperatoria de la localización de las lesiones.

de los cortes tomográficos no se corresponda exactamente con la de ninguno de los cortes del atlas, el algoritmo implementado realiza una búsqueda del corte más cercano. El usuario del sistema puede variar la distancia máxima entre ellos (Distancia máxima imagen-atlas) y adicionalmente es posible proyectar los cortes sobre imágenes no paralelas a los cortes originales del atlas (Ángulo máximo imagenatlas) (Figura 3).

Actualización. Utilizando las transformaciones descritas, el posicionamiento final del atlas se realiza en función de la información electrofisiológica obtenida mediante el registro y microestimulación de estructuras profundas en múltiples trayectorias exploratorias ${ }^{5,6}$.

\section{Resultados}

Se desarrolló un atlas digital de Schaltenbrand y Wharen, el cual se incorporó al sistema STASSIS para la planificación de neurocirugías guiadas por imágenes. Este sistema contiene múltiples herramientas de procesamiento y análisis de imágenes médicas como son: cálculo de coordenadas estereotácticas para múltiples marcos estereotácticos, medición de distancias, cálculo de volúmenes, ampliaciones, reconstrucciones $2 \mathrm{D}$ y $3 \mathrm{D}$, entre otras. La interfaz gráfica permite la visualización de los tres planos del atlas superpuestos a los respectivos cortes anatómicos, así como reportar el nombre y resaltar los contornos de una estructura cuando ésta es señalada. También es posible calcular y visualizar blancos y trayectorias y simular lesiones en las tres dimensiones.

El sistema permite la navegación continua tanto a través de los cortes originales como de las reconstrucciones. Esta facilidad, junto a la sincronización de múltiples vistas y el uso de ampliaciones, permite incrementar la exactitud en la localización de las comisuras y otros blancos, así como la selección de las trayectorias óptimas. El método implementado para el cálculo de coordenadas estereotácticas, descrito en la sección anterior, logra una exactitud submilimétrica, como ya se ha publicado por nuestro grupo ${ }^{21}$.

La localización de los blancos quirúrgicos puede ser determinada de manera directa o indirecta. En el modo directo se asignan las coordenadas estereotácticas al punto blanco visible en las imágenes. En el modo indirecto se utiliza la posición relativa del blanco con respecto a otro punto determinado previamente.

El corregistro de los contornos del atlas con los datos reales del paciente se ilustra en la figura 4 para una subtalamotomía. Una vez localizadas CA y CP es posible visualizar las secciones del atlas de SW sobre los cortes de TAC o RMN (Figura 4 A), utilizando un escalamiento lineal con respecto a las comisuras. Los contornos del atlas se ajustan a la anatomía real del paciente utilizando las 
transformaciones descritas en la sección anterior (Figura 4 B). Utilizando los datos del registro electrofisiológico, la estimulación y la exploración clínica intra-operatorios, es posible actualizar la posición del atlas en la región del blanco y simular la posición y volumen de la lesión (Figura 4 C).

El sistema permite cargar y procesar imágenes postoperatorias sin marco, provenientes de múltiples modalidades de imágenes, lo que permite evaluar la localización de las lesiones terapéuticas (Figura 4 D).

\section{Discusión}

El uso de un atlas estereotáctico digital aporta objetividad y consistencia al procedimiento neuroquirúrgico. En los casos en que los blancos quirúrgicos no son identificables en las imágenes, el atlas estereotáctico aporta una localización anatómica consistente basada en una definición estadística, por lo que esta aproximación es acertada cuando se utilizan modalidades de imágenes con bajo contraste de tejido, como la TAC.

La principal limitación del atlas de SW es que este está confeccionado a partir de un número limitado de cerebros $^{4,15,22}$, por lo que es de esperar diferencias geométricas entre aquél y la anatomía individual de los pacientes. Adicionalmente se han descrito inconsistencias en las series axial y sagital del atlas original ${ }^{10,11}$. Una posibilidad para corregir estas inconsistencias es el corregistro (matching) de cada uno de los cortes del atlas con los respectivos cortes anatómicos de RMN o TAC. Se han descrito varios algoritmos para el corregistro no lineal de atlas estereotácticos con imágenes médicas ${ }^{7,20}$. La mayoría de estos métodos consumen gran cantidad de tiempo y recursos de computadora, lo que los hace inaplicables en la práctica clínica. Los métodos más rápidos, como el propuesto por Thirion et. al. ${ }^{19}$, no son capaces de identificar con exactitud estructuras como CA, CP u otras, cuya localización exacta es indispensable en este tipo de procedimiento.

Las transformaciones geométricas descritas en este trabajo y disponibles en nuestro sistema satisfacen gran parte de la variabilidad normal entre sujetos. Adicionalmente la facilidad para registrar independientemente cada una de las series del atlas con las respectivas imágenes compensa parcialmente las inconsistencias en el atlas original. Los parámetros de transformación pueden ser modificados de forma interactiva durante el procedimiento quirúrgico, permitiendo introducir la información del registro electrofisiológico para lograr una localización más precisa del núcleo. El escalamiento se puede realizar no sólo en la dirección de la línea CA-CP, sino también en dirección dorso-ventral y lateral, permitiendo un número mayor de grados de libertad que el escalamiento unidimensional estándar. Las ventajas del escalamiento multidimensional en aplicaciones especí- ficas han sido discutidas con anterioridad ${ }^{12,23}$, y constituye otra forma de compensar las inconsistencias del atlas original.

El corregistro rígido implica que no existen distorsiones no lineales significativas en los componentes del sistema. Esta suposición es acertada en lo referente al equipamiento estereotáctico y a las imágenes de TAC. Sin embargo, este es un problema crítico cuando se utilizan imágenes de $\mathrm{RMN}^{2,3,8,16,18}$. La combinación de información procedente de varias modalidades de imágenes, atlas estereotáctico y registro electrofisiológico, es la estrategia que ofrece las mayores potencialidades para compensar las deficiencias individuales y maximizar la exactitud en la localización del blanco quirúrgico. Esta es una herramienta computacional con un amplio rango de aplicaciones en neurocirugías funcionales, tanto en procedimientos ablativos como en aquéllos que utilizan estimulación profunda (DBS). Las facilidades para actualizar la localización y dimensiones del núcleo de acuerdo a la evidencia electrofisiológica adquiere mayor relevancia en las técnicas ablativas, donde es necesario evaluar no sólo la localización del centro de la lesión, sino su extensión y las áreas que serán dañadas. Dichas transformaciones permiten crear una visión más realista de la cito-arquitectura del núcleo particular, correlacionar sobre bases estadísticas con su somatotopia, mejorar la capacidad de decisión del equipo quirúrgico y eventualmente minimizar los efectos secundarios relacionados a los procedimientos ablativos.

El sistema STASSIS constituye la herramienta de planificación para neurocirugía estereotáctica en 11 instituciones en Cuba y Chile. Hasta marzo del 2004 el atlas computarizado se ha usado exitosamente en la guía de más de 400 talamotomías, palidotomías y subtalamotomías.

El atlas de Schaltenbrand y Wharen permite la localización precisa de estructuras subcorticales ${ }^{10}$. Nuestra experiencia confirma una buena correlación entre los blancos anatómicos y funcionales. El uso de una versión electrónica contribuye a mejorar la exactitud en la localización de los blancos quirúrgicos, reduce el tiempo de planificación y del propio acto quirúrgico, y constituye una herramienta eficaz para el análisis postoperatorio de la localización de las lesiones.

\section{Bibliografía}

1. Digital Imaging and Communications in Medicine (DICOM). NEMA. National Electrical Manufacturers' Association. Rosslyn, Va: 1999. p. PS 3.5-PS 3.6.

2. Bednarz, G., Downes, B., Corn, B.W., Curran, W.J., Goldman, H.W.: Evaluation of the spatial accuracy of magnetic resonance imaging-based stereotactic target localization for gamma Knife radiosurgery of functional disorders. Neurosur- 
gery $1999 ; 45:$ 1156-1163.

3. Benardete, E.A., Leonard, M.A., Weiner, H.L.: Comparrison of frameless stereotactic systems: Accuracy, Precision and applications. Neurosurgery 2001; 49: 1409-1416.

4. Carballo, M., Rodríguez, R., López, G., Torres, A.: Sistema tridimensional de planeamiento quirúrgico para PC. Ingeniería Electrónica, Automática y comunicaciones 2005; 26: 19-23.

5. López-Flores, G., Miguel-Morales, J., Teijeiro-Amador, J. et al.: Anatomic and neurophysiological methods for the targeting and lesioning of the subthalamic nucleus: Cuban experience and review. Neurosurgery 2003 April; 52: 817-830.

6. López-Flores, G., Morales, J.M., Teijeiro, J. et al.: Localización bilateral y simetría del núcleo subtalámico. Revista de Neurologia 2003; 37: 404-412.

7. Maintz, J.B.A., Viergever, M.A.: A survey of Medical Registration. Med Image Anal 1998; 2: 1-37.

8. Michiels, J., Bosmans, H., Pelgrims, P., Vandermeulen, D., et al.: On the problem of geometric distortion in magnetic resonance images for stereotactic neurosurgery. Magn Reson Imaging 1994; 12: 749-765.

9. Montoya, A.T., Pereira, J.: Testing the precision of stereotactic planning systems. Comp Med Imaging and Graphics 1998; 22: 317-321.

10. Nieman, K., Naujokat, Ch., Phol, G., Wollner, C., Keyserlingk, D.: Verification of the Schantenbrand and Wahre Stereotactic Atlas. Acta Neurochir (Wien) 1994; 129 : $72-81$.

11. Nowinski, W.L.: Co-Registration of the SchaltenbrandWahren Microseries with the Probabilistic Functional Atlas. Stereotact Funct Neurosurg 2004 October 4; 82: 142-146.

12. Nowinski, W.L.: Anatomical targeting in functional neurosurgery by the simultaneous use of multiple Shaltenbrand-Wahren brain atlas microseries. Stereotact Funct Neurosurg 1998; 71: 103-116.

13. Nowinski, W.L., Fang, A., Nguyen, B.T. et al.: Multiple brain atlas database and atlas-based neuroimaging system. Comput Aided Surg 1997; 2: 42-66.

14. Nowinski, W.L., Yeo, T.T., Thirunavuukarasuu, A., Seng, T.T.: Microelectrode-guided functional neurosurgery assisted by clinical brain atlas CD-ROM. Comput Aided Surg 1998; 3: 115-122.

15. Schaltenbrand, G., Wahren, W.: Atlas for Stereotaxy of the Human Brain. Stuttgart: Thieme; 1977.

16. Smith, J.R., Hardy, T.L., Rose, D.F., Flanigin, H.F.: Comparison of CT-versus MRI-Guided, computer-assisted depth electrode implantation. Stereotact Funct Neurosurg 1992; 58: 189-193.

17. Sramka, M., Ruzicky, E., Novotny, M.: Computarized brain atlas in functional neurosurgery. Stereotact Funct Neurosurg 1997; 69: 93-98.

18. Sumanaweera, T.S., Adler, J.R., Napel, S., Glover, G.H.: Characterization of spatial distortion in Magnetic Resonance Imaging ang its implications for stereotactic surgery. Neurosurgery 1994; 35: 696-704.

19. Thirion, J.P.: Image matching as a diffusion process: an analogy with Maxwell's demons. Med Image Anal 1998; 2: 243-260.

20. Toga, A.W., Thompson, P.M.: The role of image registration in brain mapping. Image and Vision Computing 2001; 19: 3-24.

21. Torres, M.A., Pereira, J.: Testing the precision of stereotactic planning systems. Comput Med Imaging Graph 1998 July; 22: 317-321.

22. Vayssiere, N., Hemm, S., Cif, L. et al.: Comparison of atlas- and magnetic resonance imaging-based stereotactic targeting of the globus pallidus internus in the performance of deep brain stimulation for treatment of dystonia. J Neurosurg 2002; 96: 673-679.

23. Yeo, T.T., Nowinski, W.L.: Functional neurosurgery aided by use of an electronic brain atlas. Acta Neurochir Suppl (Wien) 1997; 68: 93-99.

24. Yu, Ch., Apuzzo, M.L., Zee, C., Petrovich, Z.: A Phantom Study of the Geometric Accuracy of Computed Tomographic and Magnetic Resonance Imaging Stereotactic Localization with the Leksell Stereotactic System. Neurosurgery $2001 ; 48$ : 1092-1099.

Carballo-Barreda, M.; Rodríguez-Rojas, F.; Torres-Montoya, A.; López-Flores, G.: Atlas computarizado para la planificación de neurocirugías estereotácticas funcionales guiadas por imágenes. Neurocirugía 2007; 18: 478-484.

Correspondencia postal: Maylén Carballo Barreda. CIREN. Ave. 25 No. 15805 entre 158 y 160. Rpto. Cubanacán, Playa. Ciudad de la Habana. Cuba. 